\section{The world's most incredible family is back}

After almost 14 years since Disney Pixar introduced us to their Incredibles family, they are back on our screens and Incredibles 2 is sure to be a hit this Christmas, so why not use the motivational power of this crime-fighting family to get your younger patients focused on fighting plaque?

Oral-B has launched a new Power Kids Electric Toothbrush (suitable from age 3+) featuring fun and friendly Incredibles 2 Disney characters. Children can download the Disney Magic Timer App by Oral-B to help them brush for the recommended

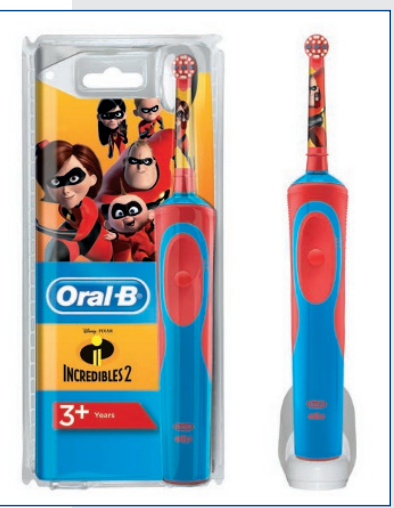
two minutes. Disney's new Incredibles 2 characters will keep them motivated and encourage proper oral care habits that will last a lifetime.

Benefits include the magical 'brush and discover' functionality. As children brush their teeth, they can reveal a secret image. The longer they brush, the more is revealed. They can also unlock fun rewards gaining stickers. Children can fill their own sticker album with stickers earned for brushing.

\section{Cloud software integration grows}

Integration with NHS services for referring patients to specialists is important and so with Dentally - the UK cloud dental software - you can refer with ease for a seamless transition of care for your patient.

Dentally has announced that it now integrates with Vantage Rego Patient Referral, a specialist dental electronic referral service (DeRS) which enables you to ensure that all of your providers are utilised in the most suitable and cost-effective way. Offering the best patient care with the shortest waiting times, Rego helps dentists select the best care for their patient by determining the most appropriate treatment services. It places and tracks the referral with complete visibility but only to those who authorised it. With Rego, the user can avoid referrals being bounced around, confident that they are being sent to the appropriate place.

Rego integration with Dentally is seamless, says the company, so all the dentist needs to do is enter their assessment in a smart form (with a built-in triage process) and Rego only asks the next relevant question to generate the suitable referral options within a matter of seconds. Once an option is chosen, the referral is placed directly with the provider. The dentist, patient and provider will all be able to trace the referral securely.

Key features of the approach are that:

- It offers the best patient care with the shortest waiting times

- Rego helps dentists select the best care for their patient by determining the most appropriate treatment services

- Integration with Dentally is seamless with quick and easy referrals

- Once an option is chosen, the referral is placed directly with the provider

- The dentist, patient and provider will all be able to trace the referral securely.

More information is available by visiting https://dentally.co.uk or calling 02038565610 .

\section{The most sophisticated secretary}

If you want to save time reporting on radiographs but still have all of the essential information to create the best possible care plan, PROPACS from PRO Diagnostics UK can help.

A one-of-a-kind online storage solution for diagnostic images, radiographs uploaded to PROPACS can be sent to a team of specialist dental radiologists for expert appraisal. Full reports will be sent back to your account within 24 hours, quickly giving you the information you need to create the best possible care plan for the patient.

Furthermore, as these images are stored directly in your PROPACS account, you can share them with authorised colleagues or technicians in order to facilitate treatment and the creation of any restorative devices. All they need to do is request free log in details and they can access the images, and the whole process is GDPR compliant and significantly reduces the chances of patient information being leaked.

Available to access anywhere, anytime, PROPACS is described as the ultimate sophisticated secretary and will streamline your diagnostic procedures like never before.

More information is available by visiting https://proxraymanagement.com/ or emailing sales@prodiagnostics.co.uk.

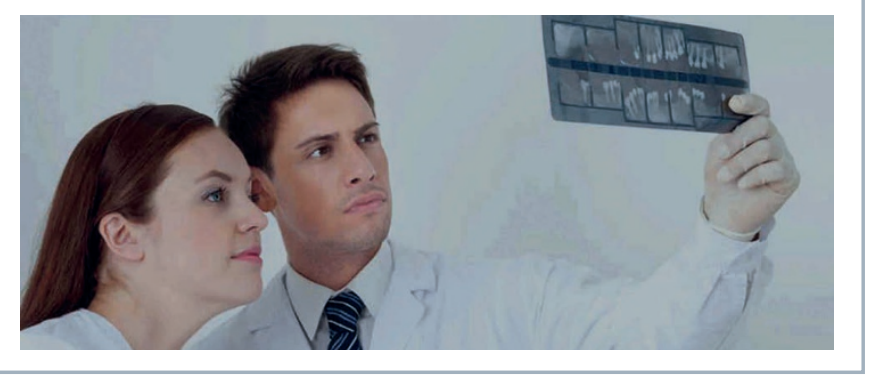

\section{Keeping ahead of the curve}

Dental implantology is fast becoming one of the most significant fields in modern dentistry and professionals can ensure they keep up-to-date with all of the latest advancements at Association of Dental Implantology (ADI) events.

Dedicated to supporting education, the ADI hosts a number of events throughout the year that will help you stay abreast of the latest news and training in the field.

Attending these will keep you ahead of the curve in dental implantology, helping you to stay at the top of your game and able to offer your patients excellent care.

Often playing host to world-renowned speakers who use these opportunities as a forum to impart their knowledge, ADI events are a chance to network within the profession and meet colleagues who share your interests.

Becoming a member of the ADI means you can benefit from free ADI Study Clubs and also receive discounted rates for other important events such as ADI Team Congresses, ADI Masterclasses and ADI Focus Meetings, all of which are geared towards helping you broaden your knowledge.

To find out more about becoming a member of the ADI and to see which events are upcoming, visit the ADI website today at www.adi.org.uk. 